\title{
Desarrollo de una herramienta computacional para el análisis de conductancia galvánica de la piel, ECG y frecuencia respiratoria por arritmia sinusal respiratoria (SCRATER)
}

\section{Development of a computational tool for the analysis of galvanic skin conductance, EKG and respiratory rate due to respiratory sinus arrhythmia (SCRATER)}

\author{
F.J. Alvarado-Rodríguez', R. Romo-Vázquez2, H. Vélez-Pérez², A.A. González-Garrido' \\ IInstituto de Neurociencias, CUCBA, Universidad de Guadalajara, Guadalajara, Jalisco, México.
}

2Depto. Ciencias Computacionales, CUCEI, Universidad de Guadalajara, Guadalajara, Jalisco, México.

\section{RESUMEN}

La existencia de una correlación entre la frecuencia cardíaca (FC), la frecuencia respiratoria (FR) y las respuestas electrodérmicas de la piel (Skin Conductance Response, SCR) ha sido reportada en la literatura, así como también el uso de estos parámetros como medida del nivel de activación del sistema nervioso autónomo. Objetivo: Este trabajo presenta una herramienta (SCRATER) para el análisis conjunto de SCR, FC y FR, las dos últimas, calculadas a partir del análisis del registro de electrocardiograma (ECG). Metodología: En esta investigación, se realizó una descripción detallada de cada algoritmo desarrollado, asi como una una descripción de la interfaz para utilizarlos. En la validación de los algoritmos empleados, se analizaron 192 registros de ECG y 231 registros de actividad electrodérmica (Electro-Dermal Activity, EDA) de 40 participantes masculinos sanos, de los cuales se calculó el número de complejos QRS y FC en cada registro de ECG y el número de SCRs de cada registro de EDA. Resultados: Los datos obtenidos fueron comparados con otras herramientas que analizan SCR y FC pero de manera independiente, obteniendo resultados equiparables mediante coeficientes de correlación. Limitaciones: El ruido y los artefactos presentes en los registros no permiten una correcta estimación de los parámetros y afectan los resultados de todas las herramientas empleadas en el desarrollo de este trabajo. Valor: SCRATER ofrece tres ventajas principales sobre las otras herramientas: 1) libre acceso, 2) código abierto y no utiliza formatos codificados o exclusivos. Conclusión: Este trabajo proporciona una herramienta computacional gratuita que permite analizar simultáneamente SCRs, FC y FR.

PALABRAS CLAVE: Herramienta computacional, conductancia galvánica, SCR, ECG, frecuencia respiratoria, frecuencia cardíaca. 


\section{ABSTRACT}

The existence of a correlation between heart rate (HR), respiratory rate (RR) and skin conductance response (SCR) has been reported in the literature, as well as the use of these parameters as a measure of the activation level of the autonomous nervous system. Objective: This paper introduces a computational tool (SCRATER) developed with the aim to analyze simultaneous recordings of SCR, and heart and respiratory rates, which were calculated from the electrocardiogram recording (ECG) analysis. Methodology: In this research, a detailed description of each developed algorithm was made, as well as a description of the interface to be used. In the validation of the algorithms used, 192 ECG records and 231 Electro-Dermal Activity (EDA) registers of 40 healthy male participants were analyzed, from which the number of QRS complexes and HR in each ECG record and the number of SCRs of each EDA record are calculated. Results: The data obtained were compared with other tools that analyze SCR and HR separately, obtaining comparable results using correlation coefficients. Limitations: The noise and artifacts present in the records do not allow a correct estimation of the parameters and affect the results of all the tools used in the development of this work. Value: SCRATER offers three main advantages over other tools: 1) free access, 2) open source and 3) does not use coded or exclusive formats. Conclusion: This work provides a free computational tool that allows simultaneous analysis of SCRs, FC and FR.

KEYWORDS: Computational tool, skin conductance resistance, ECG, respiratory rate, heart rate.

\section{Correspondencia}

DESTINATARIO: Hugo Abraham Vélez Pérez

INSTITUCIÓN: Departamento de Ciencias

Computacionales, CUCEI, Universidad de Guadalajara DIRECCIÓN: Blvd. Marcelino García Barragán \#1421, C.P. 44430, Guadalajara, Jalisco, México. CORREO ELECTRÓNICO: hugo.velezperez@gmail.com

\section{Fecha de recepción:}

14 de julio de 2016

Fecha de aceptación:

2 de septiembre de 2016 


\section{INTRODUCCIÓN}

La actividad electrodérmica ha sido ampliamente utilizada como una medida del nivel de activación del sistema nervioso autónomo (arousal), que refleja la actividad del sistema nervioso simpático ${ }^{[1]}$. Se pueden observar dos tipos generales de medidas: las medidas tónicas (Skin Conductance Level, SCL) y las fásicas (Skin Conductance Response, SCR). Las primeras se refieren a los niveles básicos en la conductancia de la piel y son un indicador del nivel arousal mientras que las segundas se refieren a los cambios breves en la actividad fisiológica tónica, los cuales están asociados a un estímulo identificado en la mayoría de los casos. En general, se considera como respuesta a toda variación en el nivel tónico superior o igual a 0.05 microsiémens $(\mu S)^{[2]}$. Las medidas de SCRs en particular han ganado popularidad en estudios clínicos, neurocientíficos y lógicos sobre emociones, toma de decisiones y condicionamiento, orientación, atención y decepción ${ }^{[3]}$. Los elementos o medidas que componen la SCR son básicamente 3: el tiempo de latencia, la intensidad y la duración de la respuesta ${ }^{[4]}$. La medición de la actividad electrodérmica (Electro-Dermal Activity, EDA) puede hacerse de manera endosomática (sin la aplicación de una corriente externa) o exosomática (con la aplicación de una corriente directa o alterna) $)^{[5]}$.

El análisis de la variabilidad de la frecuencia cardíaca es otro indicador que ha sido empleado para evaluar el estado funcional del sistema nervioso autónomo, especialmente en estadíos clínicos asociados al envejecimiento, enfermedad y estados psicológicos. Además, se ha observado una relación entre la actividad cardíaca y la frecuencia respiratoria ${ }^{[6]}$. La serie del intervalo R-R del electrocardiograma (ECG) es usada para evaluar los periodos de la frecuencia cardíaca, cuyas diferencias corresponden con los ciclos respiratorios de inhalación y exhalación. Típicamente, los periodos cardíacos disminuyen durante la inspiración e incrementan durante la expiración. Este fenómeno es llamado Arritmia Sinusal Respiratoria (Respiratory
Sinus Arrhytmia, RSA) ${ }^{[7]}$ y se considera el componente periódico más sobresaliente de variabilidad de la frecuencia cardíaca $^{[8]}$.

En la literatura se reporta la existencia de una correlación entre la frecuencia cardíaca, la frecuencia respiratoria y las respuestas electrodérmicas de la piel (conductancia) ${ }^{[1,6]}$. También existen diversas herramientas computacionales que estiman las respuestas de conductancia de la piel como SCRGAUGE[9], Ledalab ${ }^{[10]}$, SCRalyze $^{[11]}$, Autonomate ${ }^{[3]}$ y herramientas para el estudio del ECG como ECGLab ${ }^{[12]}$, Kardia ${ }^{[13]}$, Kubios ${ }^{[14]}$ o LabVIEW Biomedical toolkit ${ }^{[15]}$. Por otro lado, han sido desarrolladas algunas herramientas que permiten estimar la frecuencia cardíaca y las respuestas de conductancia de la piel de manera simultánea como son: AcqKnowledge (BIOPAC Systems Inc., Goleta, CA) y Psychlab $^{[16]}$. Sin embargo, la adquisición de este tipo de software implica un costo que en muchas ocasiones es elevado o bien, únicamente se permite el uso de formatos específicos. La herramienta propuesta en este trabajo pretende ser gratuita, de código abierto y capaz de aceptar múltiples formatos, lo que le otorga evidentes ventajas sobre el resto de los programas desarrollados con propósitos similares.

Debido a la relevancia que tiene la medición de SCL y SCR diversos trabajos en la literatura han sido reportados. Sin embargo, sólo algunos de estos trabajos refieren el uso de alguna herramienta computacional que les permita estimar las respuestas de conductancia de la piel ${ }^{[17-20]}$; mientras que el resto no reportan el uso de tal herramienta ${ }^{[21-28]}$. Evidentemente, la ausencia de una herramienta de esta naturaleza, podría conducir a errores en el conteo, impactando directamente en los resultados de la investigación. Otra posible desventaja de no contar con esta herramienta es que las señales de amplitud baja o con una definición pobre podrían ser eliminadas por el usuario, disminuyendo así el número de elementos en las bases de datos, originando pérdidas económicas y de tiempo. Es por esto que la imple- 
mentación y uso de una herramienta automática para la estimación de respuestas de SCL y SCR resulta valiosa en estudios en donde interviene la actividad electrodérmica. Lo anterior ubica a SCRATER como una importante y potencial herramienta dentro de la ingeniería biomédica nacional e internacional.

\section{METODOLOGÍA}

En este trabajo se emplearon registros adquiridos a través del método exosomático ${ }^{[5]}$, usando corriente directa debido a que es el más común. Este método se basa en la aplicación de un voltaje, en este caso $5 \mathrm{~V}$, entre dos electrodos ubicados en la superficie de la palma de la mano. En esta configuración se incluye una resistencia de valor relativamente bajo en un arreglo en serie con la piel (e.g. $1 \mathrm{k} \Omega$ ). Debido a que la resistencia de la piel es del orden de $100 k \Omega$, esta resistencia en serie afectará de manera insignificativa al flujo de corriente a través de la piel.

El voltaje es entonces constante y, por ley de Ohm, la corriente I es calculada como:

$$
I=\frac{V}{R_{p}}
$$

donde $V$ representa el voltaje (constante) y $R_{p}$ la resistencia de la piel. En términos de la conductancia $G$, la Ec. 1 puede ser representada como:

$$
I=E G_{p}
$$

De esta forma, la corriente que atraviesa la resistencia en serie será proporcional a la corriente medida y el voltaje registrado por:

$$
E=I R_{s}
$$

donde $R_{s}$ representa a la resistencia en serie y $E$ es el voltaje que corresponderá a los cambios de conductancia de la piel ${ }^{[12]}$.
Los componentes fásicos y tónicos de la actividad electrodérmica deben ser evaluados por separado ya que las SCRs son de amplitud pequeña.

\section{Base de datos}

En este trabajo se analizaron 423 registros en total (192 registros de ECG y 231 registros de EDA). Las señales estudiadas fueron obtenidas de 40 participantes masculinos sanos en 6 condiciones distintas: estado de ayuno (con 3 tareas de carga cognitiva distintas) y en estado sin ayuno (con 3 tareas de carga cognitiva distintas). El rango de edad de los participantes se encuentra entre los 19 y 35 años teniendo un promedio de 26.6 años y una desviación estándar de 5.01 años. El consentimiento informado de cada participante se obtuvo por escrito y el proyecto fue avalado previamente por el Comité de Ética del Instituto de Neurociencias de la Universidad de Guadalajara. Los registros EDA se obtuvieron por medio del método exosomático de corriente directa a $5 \mathrm{~V}$, con un equipo Bioderm 2701, que cuenta con un ADC de 12 bits con una escala de amplificación de $500 \mathrm{mV}$ por cada $0.1 \mu \mathrm{S}$. Las medidas se realizaron entre el dedo medio y el índice con electrodos de $\mathrm{Ag} / \mathrm{AgCl}$ y empleando una pasta conductora de $0.08 \mathrm{M} \mathrm{NaCl}$ a una temperatura promedio de $22^{\circ} \mathrm{C}$, y una humedad relativa del 40 al $60 \%$. Estas condiciones originan una base de datos altamente especializada permitiendo evaluar SCRATER en distintas circunstancias.

\section{Descripción de herramienta computacional}

En conjunción con el algoritmo desarrollado para la detección de SCRs, el cual se describe a continuación, se implementó una interfaz en Matlab R con el objetivo de visualizar las señales de conductancia tanto SCL como SCR. Los resultados de este análisis preliminar pueden ser exportados a Excel $\mathrm{R}$ ofreciendo así al usuario otra opción para gestionar o procesar las bases de datos generadas. La Figura 1 muestra una descripción simplificada de los módulos que integran SCRATER. 


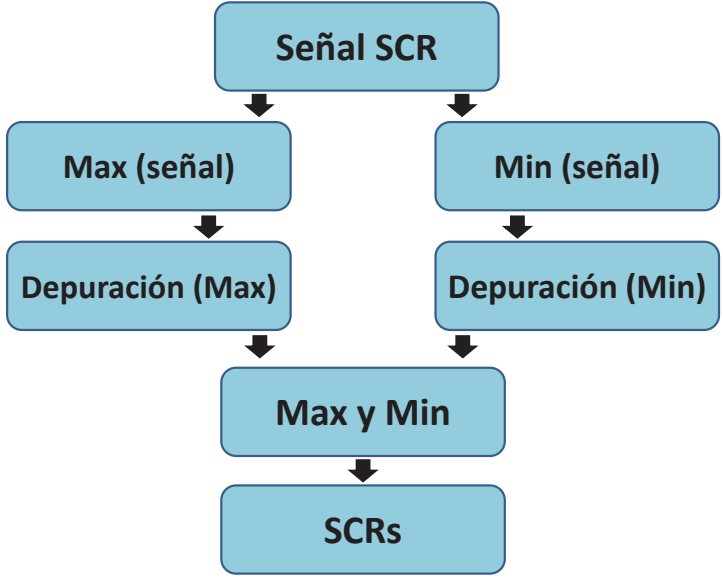

FIGURA 1: Descripción de módulos de SCRATER.

\section{Algoritmo de detección de SCRs}

Durante el registro de la conductancia de la piel se obtienen dos valores principales, el SCL y el SCR. El análisis de estos dos parámetros se puede hacer de manera completa o parcial si se realiza un paso previo de preprocesamiento con el objetivo de eliminar artefactos y ruido de los registros. El algoritmo desarrollado para la detección de SCRs en el registro de SCR se describe a continuación:

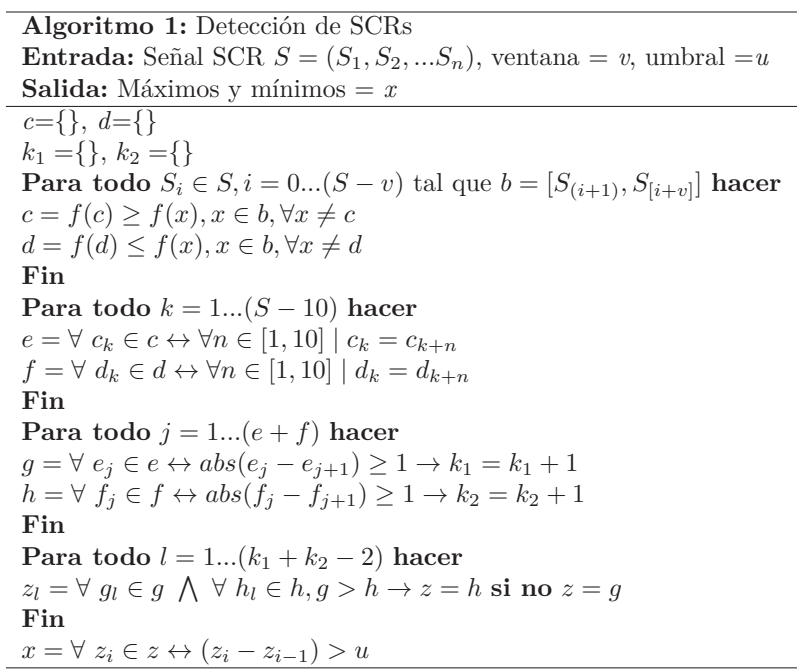

El algoritmo inicia buscando en la señal los picos máximos y mínimos absolutos en segmentos previamente definidos. Estos datos se guardan en vectores distintos (uno de máximos y el otro de mínimos). A partir de los valores guardados se realiza la eliminación de los datos repetidos, almacenando sólo los más repetidos, los cuales corresponderían a los picos y valles más representativos de la señal que se está analizando. Para evitar datos redundantes que generen conflicto durante la búsqueda automática de picos de respuestas, se implementó una segunda depuración de datos repetidos. Esta metodología permite reducir la cantidad de datos del registro de varios millares a un centenar o incluso decenas de datos que contienen los puntos máximos y mínimos de la señal. A continuación, se concatenan ambos vectores (valles y picos) para buscar las SCRs a partir de los parámetros que se designen como umbral, sin necesidad de aplicar un filtro que afecte la naturaleza de los datos y obtener así las SCRs. En la Figura 2 se puede observar un diagrama a bloques del Algoritmo 1.

\section{Algoritmo detector de complejos QRS}

Esta herramienta tiene por objetivo detectar los complejos QRS del ECG. Para esto, el algoritmo estima un factor a partir del promedio de los picos máximos y de los picos mínimos en ventanas de $1 \mathrm{~s}$, dentro de los primeros $15 s$ de cada registro de ECG.

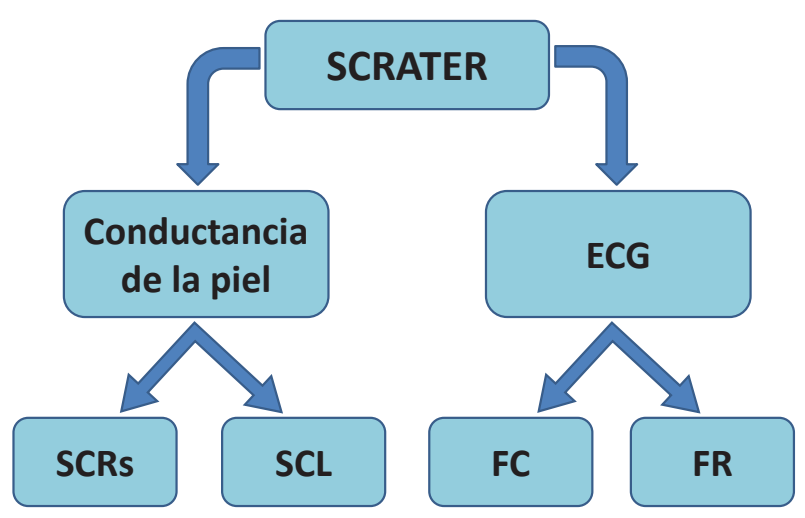

FIGURA 2: Algoritmo de detección de SCRs. 
Este factor representa la amplitud del complejo QRS y se emplea un valor entre el 30\% y 50\% del mismo para buscar valores similares en periodos de $10 \mathrm{~ms}$ a lo largo del registro de ECG. De este modo se estiman tanto la cantidad de pulsos cardíacos como su frecuencia media por minuto. El algoritmo desarrollado para la de detección de complejos QRS en el registro de ECG se describe enseguida:

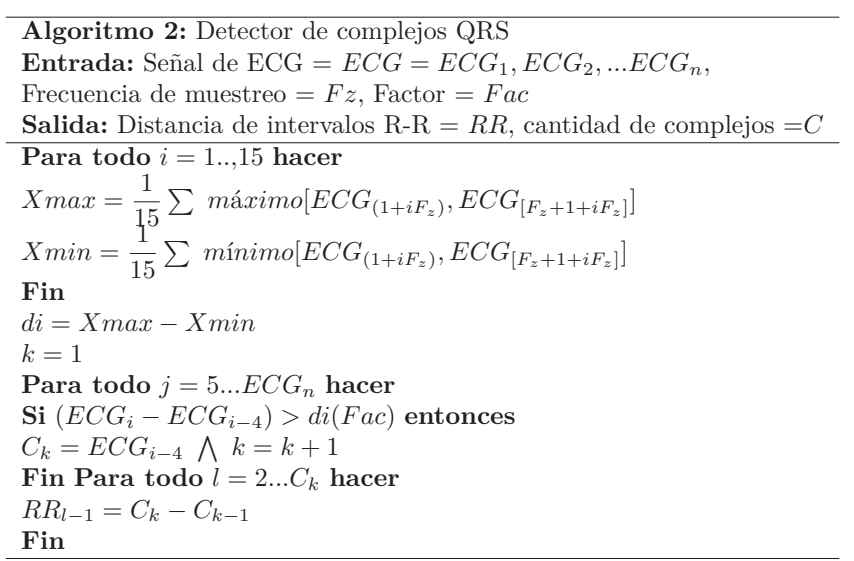

\section{Frecuencia respiratoria}

La frecuencia respiratoria es estimada a partir del ECG, específicamente de la medición de los intervalos $\mathrm{R}-\mathrm{R}$ de los registros. Estos intervalos se modifican durante la respiración, aumentando y disminuyendo su duración, haciéndose más largos durante la exhalación y más cortos durante la inhalación ${ }^{[7]}$. De esta forma es posible entonces detectar las curvas de inhalación y exhalación y así contar cada una de ellas mediante la graficación de la duración los intervalos R-R e implementación de las ecuaciones propuestas a continuación.

Si se cumple:

$\left[F r_{i}-F r_{i-1}\right] \geq 0 \wedge\left[F r_{i-1}-F r_{i-2}\right]<0$

entonces:

$$
\mathrm{Cr}=\mathrm{Cr}+1
$$

Se considerará como una curva de respiración, donde $\mathrm{Fr}$ representa las magnitudes de los intervalos R-R y $\mathrm{Cr}$ el número de curvas respiratorias detectadas. Con lo anterior como base, la frecuencia respiratoria se calcularía entonces como:

$$
\frac{C r}{\min }
$$

donde min depende de la duración del registro analizado. Aunque no es tan precisa como la medición directa del flujo respiratorio, el cálculo de la frecuencia respiratoria a partir del ECG puede ser considerada como una estimación representativa de la frecuencia respiratoria ${ }^{[14]}$.

\section{Interfaz}

La Figura 3 muestra la ventana principal de la herramienta propuesta. Como se puede apreciar, cuenta con dos secciones de análisis en las que se pueden visualizar las señales de conductancia, ECG y FR.

En la sección de conductancia, representada en la sección A de la Figura 3, la herramienta permite la modificación de tres parámetros de acuerdo a las necesidades del usuario para un mejor análisis de las SCRs:

1. El umbral de respuesta. Aunque en la mayoría de los reportes especializados en la literatura los valores típicos para el umbral se encuentran entre 0.02 y $0.05 \mu S$, el valor puede ser modificado por el usuario de acuerdo a sus necesidades y criterio, para así determinar a partir de qué valor se considerará como una respuesta.

2. La ventana de análisis. Esta sirve para discriminar respuestas demasiado cortas o falsos positivos.

3. Segmento de análisis del registro. Este permite poder eliminar segmentos que contienen falsos positivos, principalmente al inicio y al final del registro. 


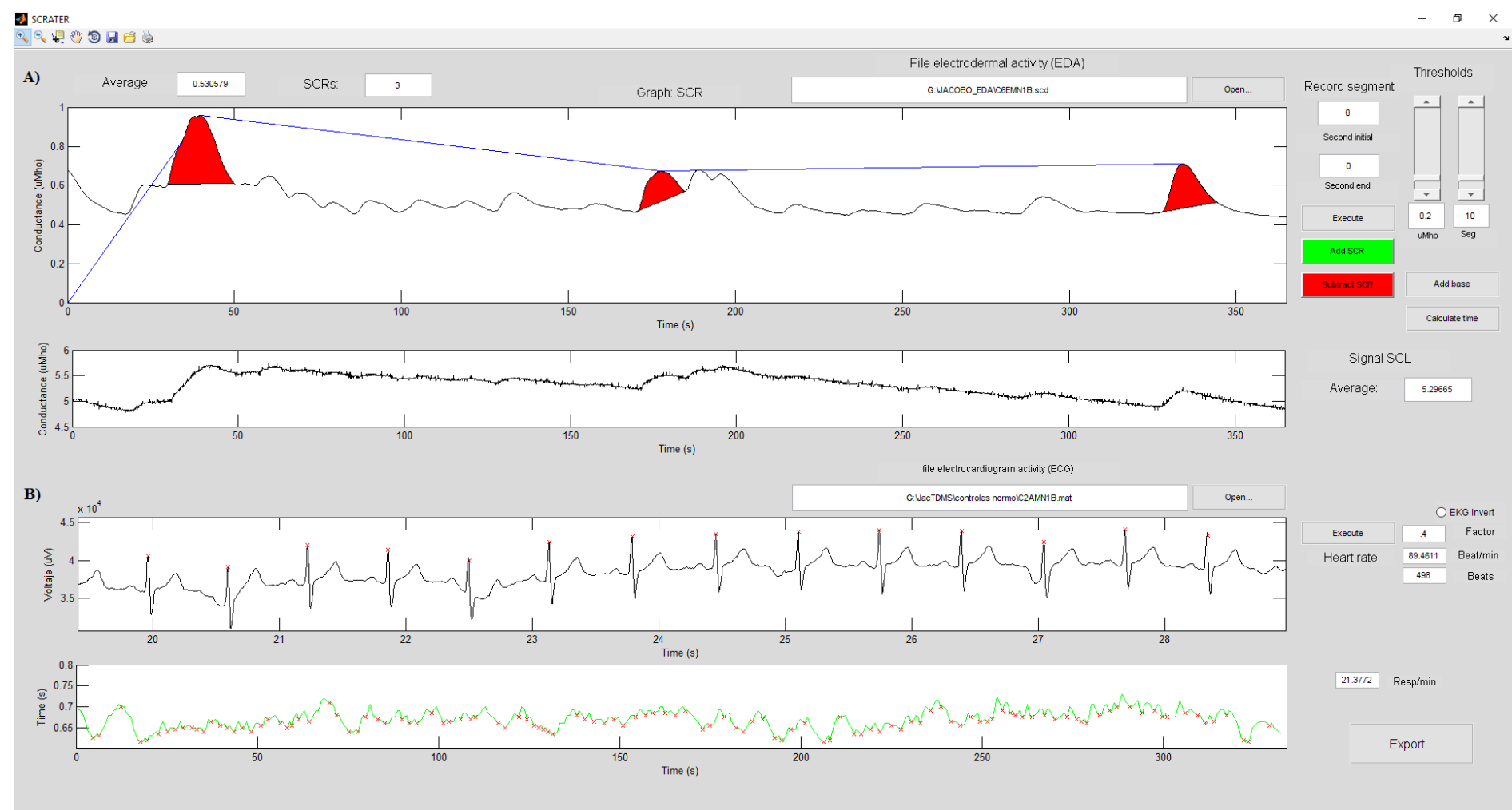

FIGURA 3: Interfaz de SCRATER. La sección A) muestra la parte de análisis de conductancia galvánica de la piel y la sección B) la parte de análisis de ECG.

Una vez efectuado el análisis automático de respuestas basado en los parámetros definidos por el usuario, se deben definir manualmente el inicio y el término de cada una de las SCRs en las que, de acuerdo a los criterios del usuario, se haya considerado que existe una respuesta. De esta manera pueden calcularse la duración de cada respuesta, su intensidad, el tiempo de elevación y el tiempo de recuperación media. Dada la variabilidad que puede tener la respuesta de conductancia y el comportamiento de su registro, se incluyó una función para agregar o eliminar manualmente picos de respuestas independientemente de su localización automática. En cuanto a la SCL, el principal parámetro calculado es el promedio del registro analizado.

La sección B de la Figura 3 está enfocada en el análisis del ECG. En esta parte se estima el número de latidos y la frecuencia cardíaca media durante el registro. La herramienta computacional cuenta con una función de ECG invertido que cambia el algoritmo de detección de complejos $Q R S$, pues en ciertos registros, dependiendo de la derivación empleada, el complejo $Q R S$ queda invertido respecto a la típica onda de ECG lo cual permite un correcto análisis de la frecuencia cardíaca. También incluye un factor de cálculo para adaptar el algoritmo de mejor manera a las características propias del registro de ECG. La estimación de la frecuencia respiratoria se calcula a partir de las distancias entre cada complejo QRS. Esta señal genera curvas en cada ciclo respiratorio y cada una de estas curvas es considerada como una respiración.

En la sección de análisis de los resultados SCRATER genera un archivo de Excel con los resultados de los análisis realizados para poder tratar los datos en diversos formatos y realizar los análisis estadísticos que se consideren pertinentes. Los resultados también pueden ser visualizados en la ventana de comandos de Matlab. En la Tabla 1 pueden observarse todos los parámetros que calcula la herramienta desarrollada. 


\section{RESULTADOS Y DISCUSIÓN}

Los resultados de los parámetros que se obtuvieron con SCRATER (Tabla 1) se compararon con Ledalab ${ }^{[10]}$ y LabVIEW Biomedical toolkit ${ }^{[15]}$. Con la finalidad de validar nuestra herramienta computacional, se empleó el coeficiente de correlación como indicador de comparación principal entre los resultados. En la Tabla 2 se agru- paron las varianzas, promedio y mediana de los resultados de cada uno de los programas empleados, en los que se calculó la cantidad de SCRs, número de complejos QRS y frecuencia cardíaca durante todo el registro analizado, para conocer la dispersión de los datos generados y observar el comportamiento de los mismos.

TABLA 1: Parámetros calculados por interfaz de SCRATER.

\begin{tabular}{|c||c|c|c|}
\hline & Parámetro & Unidad & Descripción \\
\hline \hline \multirow{4}{*}{ Conductancia } & Promedio SCR & $\mu \mathrm{S}$ & Promedio de señal SCR. \\
& Promedio SCL & $\mu \mathrm{S}$ & Promedio de señal SCL. \\
& SCRs & - & Número de respuestas dérmicas. \\
& Tstart & $\mathrm{s}$ & Tiempo de inicio de respuesta SCR. \\
& Trise & $\mathrm{s}$ & Tiempo en llegar la SCR a su pico. \\
& Duration & $\mathrm{s}$ & Duración total de la SCR. \\
& Peak & $\mathrm{s}$ & Amplitud de la SCR respecto al \\
& & & punto medio del valor de su base. \\
\hline \hline \multirow{3}{*}{ ECG } & Beat/min & $1 / \mathrm{min}$ & Número de latidos por minuto. \\
& Beats & - & Total de latidos del registro. \\
& Resp/min & $1 / \mathrm{min}$ & Frecuencia respiratoria estimada. \\
\hline \hline
\end{tabular}

La gráfica de los resultados de SCRs obtenidos en Ledalab y SCRATER de 3 diferentes umbrales equivalentes para considerar una respuesta SCR se observan en la Figura 4, la cual muestra un comportamiento similar de acuerdo a índices de correlación de hasta 0.9408 .

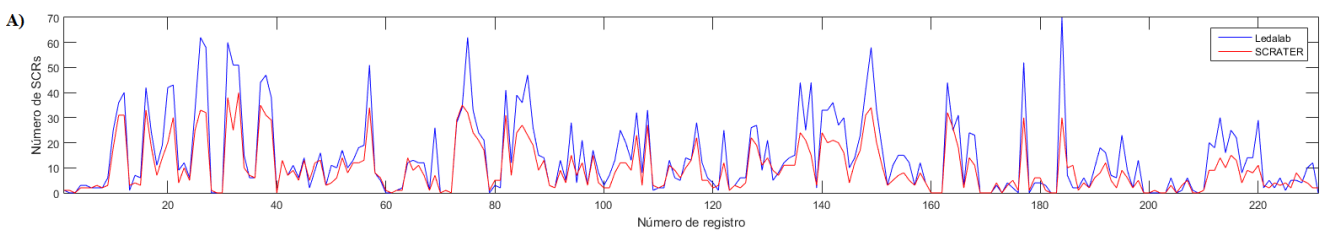

B)
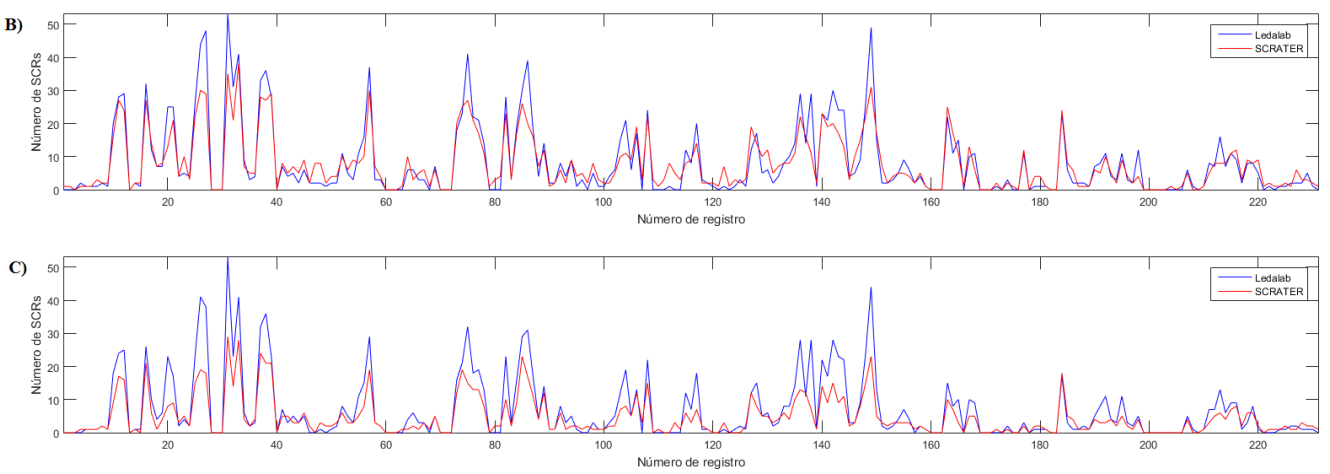

FIGURA 4: SCRs encontradas, Ledalab en azul y SCRATER en rojo. A) Coeficiente de correlación de 0.9373 con umbral de $0.06 \mu \mathrm{S}$, con una covarianza de 139.89. B) Coeficiente de correlación de 0.9371 con umbral de $0.1 \mu \mathrm{S}$, covarianza de 85.11. C) Coeficiente de correlación 0.9408, con umbral de $0.2 \mu \mathrm{S}$, covarianza de 54.19. 
Asimismo, la dispersión de los resultados observada tanto para Ledalab como para SCRATER demostró comportamientos homólogos entre ambas herramientas como lo muestra la Figura 5.
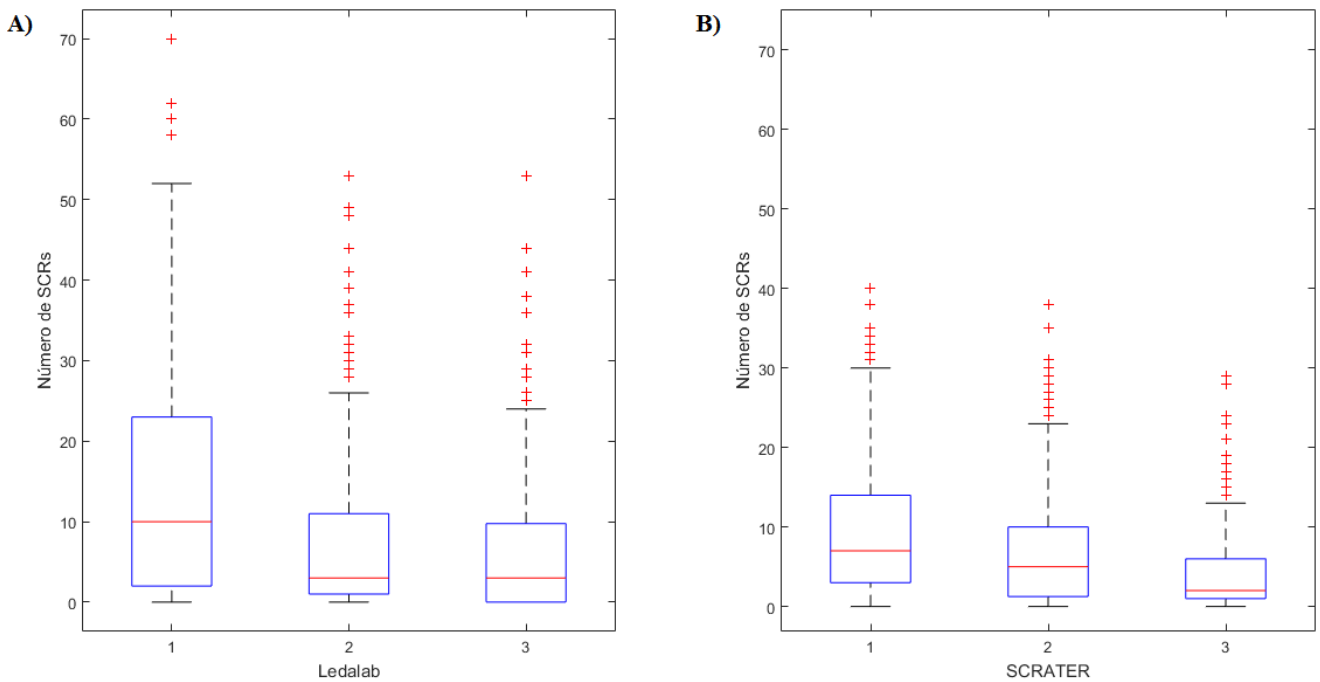

FIGURA 5: Análisis de dispersión de resultados de SCRs. En el lado A) se observan la dispersión de los resultados obtenidos con Ledalab y los tres umbrales empleados de 1) 100 unidades de medida (UM) adimensionales, 2) 200 UM y 3) 245 UM como umbral. En el lado B) la dispersión de los resultados obtenidos con SCRATER y los tres umbrales empleados de 1) $0.06 \mu \mathrm{S}, 2) 0.1 \mu \mathrm{S}$ y 3) $0.2 \mu \mathrm{S}$ como umbral.

Respecto a la etapa de análisis de respuesta de conductancia de piel, los registros de EDA que mostraron mayor diferencia en el análisis de SCRs detectadas fueron aquellos que mostraban mayor actividad (considerándose como una mayor oscilación de la señal de su estado basal) y posibles respuestas superpuestas. Estas diferencias son debidas al algoritmo empleado y la consideración de respuestas sobrepuestas o no en la señal de SCR debido a los parámetros establecidos como umbrales durante los análisis.

La frecuencia cardíaca entre LabVIEW Biomedical toolkit y SCRATER refleja un coeficiente de correlación de 0.9837 y la cantidad de complejos QRS detectados tiene una correlación de 0.9963. Se obtuvieron covarianzas entre los resultados de ambos programas de 99.0201 en el cálculo de frecuencia cardíaca y 4146.4 en conteo de complejos QRS como lo muestran los resultados de la Figura 6.
La dispersión de los resultados mostrada en la Figura 7 confirma la efectividad del algoritmo empleado en el análisis de la frecuencia cardíaca en SCRATER, que exhibe incluso una menor varianza que su contraparte comercial (Tabla 2). 

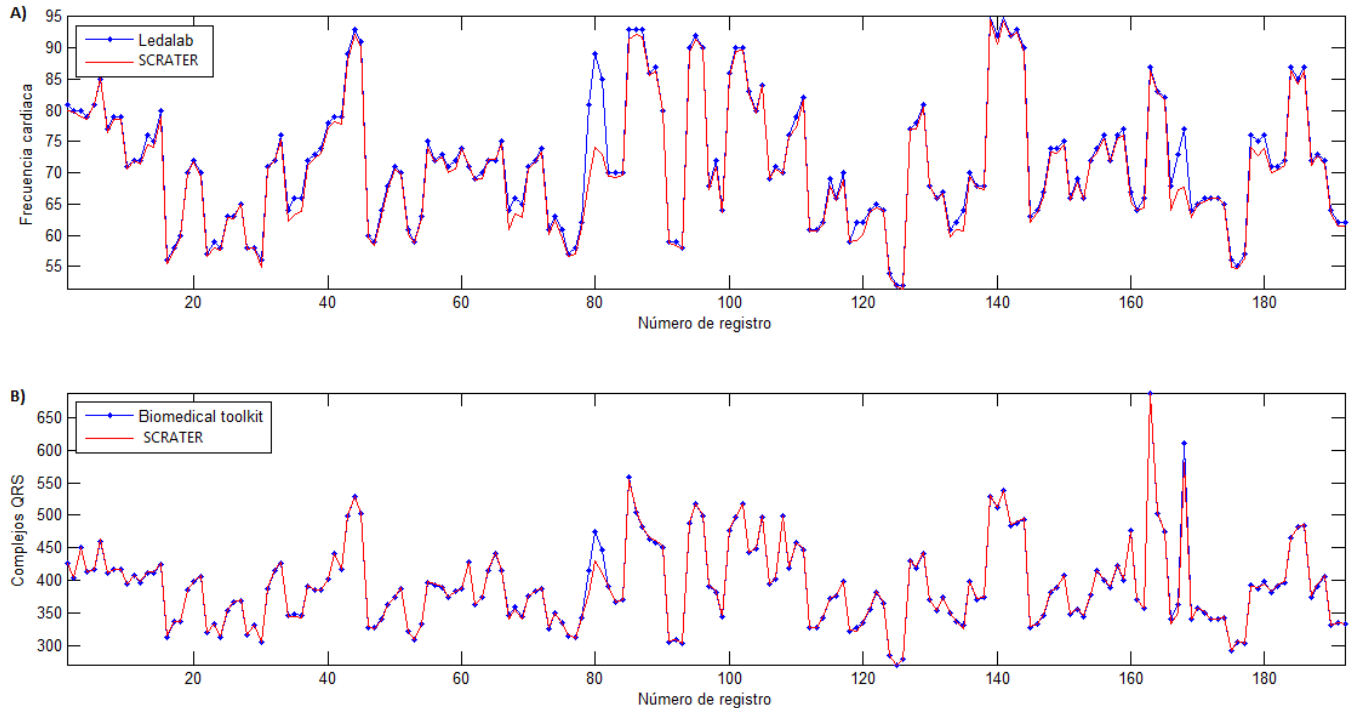

FIGURA 6: Resultados de análisis de ECG, en azul Labwiev y en rojo SCRATER. A) Gráfica que muestra la frecuencia cardíaca media. B) Cantidad de latidos por minuto detectados en cada registro.
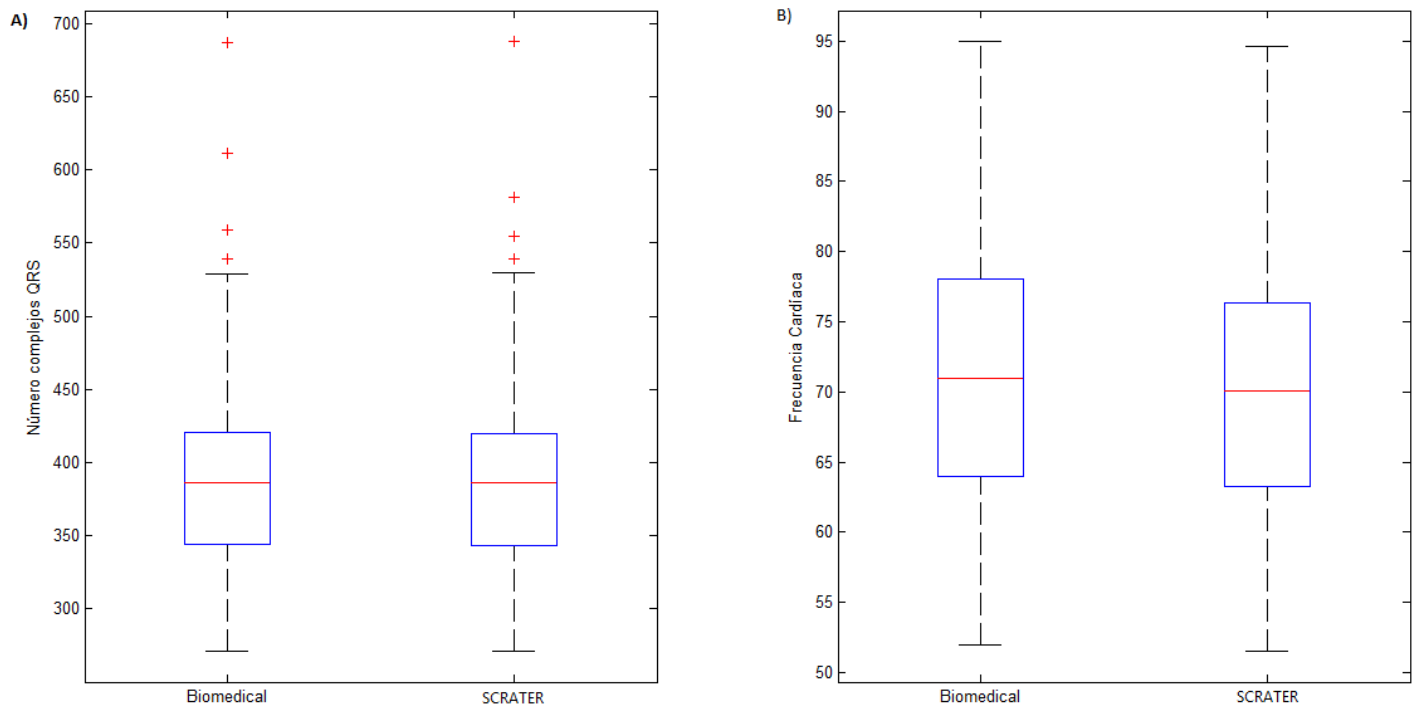

FIGURA 7: Análisis de dispersión de resultados de ECG. La sección A) muestra la dispersión de la cantidad de complejos QRS detectados y la sección B) la dispersión de las frecuencias cardíacas de los registros analizados.

Vale la pena mencionar que los registros que presentan ruido y artefactos no permiten una correcta estimación y afectan los resultados de las 3 herramientas, por lo que una mejora para la herramienta desarrollada sería agregar una parte de preprocesamiento para eliminar ruido de las señales que se encuentren muy contaminadas. 


\section{CONCLUSIONES}

Diversos estudios han demostrado una correlación funcional entre distintas variables de origen autonómicas como la actividad electrodérmica, la frecuencia cardíaca y la frecuencia respiratoria. En este contexto, se han desarrollado varias herramientas computacionales con el fin de estudiarlas y cuantificarlas pero adolecen de limitaciones principales: su costo, y el tipo de formato de aceptación de datos, usualmente limitado o exclusivo de determinada marca comercial.

Este trabajo presenta una herramienta computacional gratuita (SCRATER) que permite analizar simultánea-mente la conductancia galvánica de la piel, la frecuencia cardíaca y la frecuencia respiratoria, medidas estas dos últimas a partir de registros de ECG.
Los obtenidos por la herramienta presentada fueron comparados contra los valores estimados por 2 herramientas (Ledalab y Biomedical Toolkit de Labview) y tanto los análisis visuales como los cuantitativos presentados en este trabajo demuestran que los resultados obtenidos por SCRATER resultan equiparables a los estimados por las herramientas mencionadas.

SCRATER ofrece tres ventajas principales sobre las otras herramientas: 1) libre acceso, que permite un uso amplio e irrestricto; 2) código abierto, permitiendo modificaciones libres al código por parte de los usuarios lo que deriva en una retroalimentación y mejora continua del programa y 3) SCRATER no utiliza formatos codificados o exclusivos, permitiendo así la libre importación de datos provenientes de diversos equipos comerciales y formatos.

TABLA2: Análisis de resultados obtenidos.

\begin{tabular}{|c||c|c|c|c|c|}
\hline Herramienta & Umbral & Medida & Varianza & Promedio & Mediana \\
\hline \hline Ledalab & 100 UM & SCRs & 243.91 & 14.46 & 10 \\
& 200 UM & SCRs & 120.50 & 8.19 & 3 \\
& 245 UM & SCRs & 97.48 & 6.95 & 3 \\
\hline \hline SCRATER & $0.06 \mu \mathrm{S}$ & SCRs & 94.81 & 9.94 & 7 \\
& $0.1 \mu \mathrm{S}$ & SCRs & 68.45 & 7.65 & 5 \\
& $0.2 \mu \mathrm{S}$ & SCRs & 34.03 & 4.63 & 2 \\
\hline \hline Labview & - & FC & 101.83 & 71.69 & 71 \\
& - & QRS & 4194.60 & 393.50 & 386.5 \\
\hline \hline CRATER & - & FC & 99.51 & 70.76 & 70.05 \\
& - & QRS & 4129.00 & 393.09 & 386 \\
\hline \hline
\end{tabular}

\section{RECONOCIMIENTOS}

Se agradece al Laboratorio de Neurofisiología Clínica de la Universidad de Guadalajara por facilitar las bases de datos para probar esta toolbox. Este trabajo fue respaldado por los doctores del Centro Universitario de Ciencias Exactas e Ingenierías (CUCEI) de la Universidad de Guadalajara (UDG). 


\section{REFERENCIAS}

[1] G. Wallin. "Sympathetic Nerve Activity Underlying Electrodermal and Cardiovascular Reactions in Man," Psychophysiology, vol. 18, no. 4, pp. 470-476, 1981. DOI: 10.1111/j.1469-8986.1981. tb02483.x

[2] F. Lapuente, J. Amor, J. Selva. "Procedimientos de registro, medida y cuantificación de la actividad electrodérmica," Anales de psicología , no. 2, pp. 3-20, 1985. http://revistas.um.es/analesps/article/viewFile /215801/170021

[3] S. Green, P. Kragel, M. Fecteau, K. LaBar. "Development and validation of an unsupervised scoring system (Autonomate) for skin conductance response analysis," International Journal of Psychophysiology, vol. 91, no. 3, pp. 186-193, 2014. DOl: 10.1016/i. ijpsycho.2013. 10.015

[4] D. Levinson, R. Edelberg. "Scoring Criteria for Response Latency and Habituacion in electrodermal research: A critique," Psychophysiology, vol. 22, no. 4, pp 417-426, 1985. DOI: 10.1111/j.1469-8986.1985.tb 01626.x

[5] W. Boucsein, D. Fowles, S. Grimnes, G. Ben-Shakhar, W. Roth, M. Dawson, D. Filion. "Publication recommendations for electrodermal measurements," Psychophysiology, vol. 49, pp. 1017-1034, 2012. DOI: 10.1111/i.1469-8986.2012.01384.X

[6] G. Macefield, G. Wallin. "The discharge behavior of single sympathetic neurons supplying human sweat glands," Journal of the Autonomic Nervous System, vol. 61, no. 3, pp. 277-286, 1996. DOI: 10.1016/S0165-1838(96)00095-1

[7] P. Grossman and K. Wientjes. "Respiratory sinus arrhythmia and parasympathetic cardiac control: some basic issues concerning quantification, applications and implications," in Cardiorespiratory and Cardiosomatic Psycophysiology, pp. 117-138, 1986. DOI: 10.1007/978-1-4757-0360-3_8

[8] G. Berntson, J. Bigger, D. Eckberg, P. Grossman, P. Kaufmann, M. Malik, et al., "Heart rate variability: Origins, methods, and interpretive caveats,” Psychophysiology, vol. 34, pp. 623-648, 1997.

[9] W. Boucsein. "Electrodermal activity,” Springer Science \& Business Media, 2012.

[10] M. Benedek, C. Kaembach. "Decomposition of skin conductance data by means of nonnegative deconvolution," Psychophysiology, vol. 47, no. 4, pp. 647-658, 2010. DOl: 10.1111/j.1469-8986.2009.00972.x

[11] D. Bach, G. Flandin, K. Friston, R. Dolan. "Time-series analysis for rapid evento-related skin conductance responses," Journal of Neuroscience Methods, vol. 184, no. 2, pp. 224-234, 2009. DOI: 10.1016/i.jneumeth.20 09.08.005
[12] J. De Carvalho, A. da Rocha, F. de Olveira, J. Souza, L. Junqueira. "Development of a Matlab Software for Analysis of Heart Rate Variability," en Signal Processing 2002 6th International Conference on, IEEE, vol. 2, pp. 1488-1491, 2002. DOI: 10.1109/ICOSP.2002. 1180076

[13] P. Perakakis, M. Joffilfy, M. Taylor, P. Guerra, J. Vila. "KARDIA: A Matlab Software for the analysis of interbeat intervals," Computer Methods and Programs in Biomedicine, vol. 98, no. 1, pp. 83-89, 2010. DOI: 10.1016/j.cmpb.2009.10.002

[14] M. Tarvainen, J. Niskanen, J. Lipponen, P. Ranta-Aho, P. Karjalainen. "Kubios HRV- Heart Rate Variability Analysis Software," Computer Methods and Programs in Biomedicine, vol. 113, no. 1, pp. 210-220, 2014. DOI: 10.1016/i.cmpb.2013.07.024

[15] S. Jain, P. Kumar. "LABVIEW based expert system for detection of heart abnormalities," Advances in Electrical Engineering, pp. 1-5, 2014. DOI: 10.1109/ICAEE.2014.6838451

[16] Psychlab. Disponible en http:// www.psychlab.com, consultado el 15 de Junio del 2016.

[17] L. Carbonnell, F. Vidal, H. Sequeira, J. Caverni. "A reasoning bias revealed by electrodermal activity," Psychophysiology, vol. 43, pp. 387-393, 2006. DOI: 10.1111/j.1469-8986.2006.004 13.x

[18] M. Forgiarini, M. Gallucci, A. Maravita. "Racism and the empathy for pain on our skin," Frontiers in Psychology, vol. 2, pp. 1-7, 2011. DOI: 10.3389/fpsyg.2011.00108

[19] M. van Dooren, J. de Vries, J. Janssen. "Emotional sweating across the body: Comparing 16 different skin conductance measurement locations," Physiology \& behavior, vol. 106, no. 2, pp. 298-304, 2012. DOI: 10.1016/j.physbeh.2012.01.020

[20] C. Mackersie, N. Calderon-Moultrie. "Autonomic nervous system reactivity during speech repetition tasks: heart rate variability and skin conductance," Ear and Hearing, vol. 37, pp. 118S-125S, 2016. DOI: 10.1097/AUD.0000000000000305

[21] R. Soussignan. "Duchenne smile, emotional experience, and autonomic reactivity: a test of the facial feedback hypothesis," Emotion, vol. 2, no. 1, pp. 52-74, 2002. DOI: 10.1037/1528-3542.2.1.52

[22] D. Adolph, S. Schlösser, M. Hawighorst, B. Pause. "Chemosensory signals of competition increase the skin conductance response in humans," Physiology \& behavior, vol. 101, no. 5, pp. 666-671, 2010. DOI: 10.1016/i.physbeh.2010.08.004

[23] A. Breska, K. Maoz, G. Ben-Shakhar. "Interstimulus intervals for skin conductance response measurement," Psychophysiology, vol. 48, no. 4, pp. 437-440, 2011. DOI: 10.1111/j.1 4698986.2010.01084.x

[24] M. Naji, N. Hassanzadeh, H. Esmaeilpour Marandi. "Arousal and activation in a pistol shooting task," Journal Mil Med, vol. 12, no. 4, pp. 185-190, 2012. http://www.militarymedj. ir/article-1-723-en.pd 